\title{
UNIDAD LOGÍSTICA DE RECUPERACIŌN DE RESIDUOS DE CONSTRUCCIÓN Y DEMOLICIÓN: ESTUDIO DE CASO BOGOTÁ D.C.
}

\section{LOGISTIC UNIT TO THE RECOVERY OF CONSTRUCTION AND DEMOLITION WASTE: Study Case BOGOTÁ D.C.}

\author{
Álvaro Chávez Porras \\ Ing. Industrial, Ph.D., Profesor Asistente, Facultad de Ingeniería, \\ Líder Grupo Producción, Innovación y Tecnología, \\ Universidad Militar Nueva Granada, Bogotá, Colombia. \\ alvaro.chavez@unimilitar.edu.co \\ Óscar Palacio León \\ Ing. Industrial, M.Sc., Profesor Asistente, Facultad de Ingeniería, \\ Investigador Grupo Producción, Innovación y Tecnología, \\ Universidad Militar Nueva Granada, Bogotá, Colombia. \\ oscar.palacio@unimilitar.edu.co \\ Nataly Lorena Guarín Cortés \\ Ing. Industrial, Investigador Grupo Producción, Innovación y Tecnología, \\ Facultad de Ingeniería, Universidad Militar Nueva Granada, Bogotá, Colombia. \\ nataly.guarin@unimilitar.edu.co
}

Fecha de recepción: 28 de enero de 2013

Fecha de aceptación: 3 de febrero de 2014

\section{RESUMEN}

Uno de los problemas de los centros urbanos es la generación de "Residuos en la construcción, demolición, rehabilitación y remodelación - RCD" el cual empeora por la gestión y control dada para la correcta disposición final de éstos; además, por el poco uso potencial de reincorporación en las cadenas productivas, generando impactos socio-ambientales y económicos; caso específico de la ciudad de Bogotá D.C. Luego de la cuantificación, caracterización y análisis de los residuos generados In Situ, en el área de influencia, apoyados de tendencias y herramientas como el diseño de sistemas productivos y el concepto de aplicación en ingeniería de la ecoeficiencia, se obtuvo un diagnóstico, a través de la investigación, que permitió estructurar la proposición de un proceso de reciclaje y la identificación de recursos básicos de funcionamiento; además de la estimación y proyección de las tecnologías necesarias. Se formuló una propuesta logística con cantidades potenciales de 
los materiales a gestionar, con parámetros a nivel internacional y normatividad nacional, generando una distribución en planta piloto que cumple con las medidas de diseño de las unidades de trabajo necesarias para la futura Unidad de manejo. En general, con el modelo de gestión, se atendió lo estipulado en el Plan de Ordenamiento Territorial - POT, respecto a su ubicación y razón social, profundizado en la ecoeficiente, en conjunto con el sistema logístico, se planteó la pregunta de investigación, que identificó los recursos esenciales para una futura "Unidad Logística de Reciclaje Piloto de RCD para Bogotá D.C.."

Palabras Clave: RCD, Logística, Procesos, Reciclaje, Unidad Recuperadora.

\section{ABSTRACT}

One of the problems of the urban centers is the generation of "waste in the construction, demolition, rehabilitation and refurbishment - RCD"; which worsens by management and given control to the correct final disposal In addition, by the little potential use of reinstatement in the productive chains, generating impacts socio-environmental and economic; specific case of the city of Bogotá D.C. After the quantification, characterization and analysis of the waste generated In Situ, in the area of influence, supported trends and tools such as the design of production systems and the application in engineering of the eco-efficiency concept, obtained a diagnosis, through research, that allowed to structure the proposal of a recycling process and the identification of basic operational resources; In addition to the estimation and projection of the necessary technologies. Proposed potential quantities of materials logistics was formulated to manage, with parameters to international and national regulations, generating a distribution in pilot plant that complies with measures of design of the work units needed for the future management unit. In general, with the management model, provisions was attended the "Plan de Ordenamiento Territorial - POT" with respect to its location and social, in-depth reason in the eco-efficient, in conjunction with the logistics system, has raised the question of research, which identified the resources essential for a future "logistic unit of pilot recycling of RCD to Bogotá D.C.".

Key Words: CDW, Logistics, Processing, Recycling, Recovery Unit.

\section{INTRODUCCIÓN}

A nivel global, los principales centros urbanos y ciudades se caracterizan por el dinamismo estructural y el desarrollo ur- banístico, promovido por la esteticidad y optimización de la funcionalidad de sus componentes; lo que motiva a la construcción, rehabilitación, demolición o remodelación de sus elementos estructurales. Estos 
procesos, en muchos casos desordenados, de urbanización hasta su etapa final, han causado transformación del paisaje por su mala disposición. Lo que ha generado: Pérdida de biodiversidad y reducción del espacio público, por su aglomeración no proyectada, disminución de la calidad del aire, alteración de la oferta y calidad del recurso hídrico, pérdida y afectación de la disponibilidad de suelo; tanto como el aumento de la vulnerabilidad y generación de fragmentos y restos de materiales naturales y prefabricados. [1]

En general, los residuos generados en las fases de la construcción, Residuos de Construcción y Demolición - RCD, conocidos como "Escombros" por la comunidad, son materiales inertes generados en todas y cada una de las etapas por las que pasa una obra, a lo largo de su vida útil (alrededor de cincuenta años); constituyendo cerca del $60 \%$ de la cantidad total de los residuos generados en las ciudades [2], los cuales al aumentar su cantidad, aproximadamente, con una tasa de tendencia de $4 \%$, desencadenan diversos impactos negativos para el ambiente y la sociedad [3]. Su inadecuada gestión por los centros urbanos ha sido objeto de análisis a nivel nacional e internacional; efectuándose soluciones como el diseño y la implementación de sistemas productivos, plantas o unidades de reciclaje, que han permitido considerarlos como potencial materia prima de reincorporación a las cadenas productivas, con la obtención de agregados reciclados; lográndose a través de modelos logísticos de gestión ecoeficiente, en conjunto con sistemas produc- tivos, dando una solución económica a las regiones. En los modelos se ha requerido de niveles de desarrollo e implementación de tecnologías de reciclaje o transformación, manifiesto en el destino final, haciendo injerencia a razones de índole ambiental, económica, política y cultural. [4]

Para el caso de la ciudad de Bogotá D.C.Colombia, se presentó el contexto de los RCD generados In Situ, estudiándose, bajo un diagnóstico que los cuantificó, caracterizó y analizó; apoyados de las tendencias y herramientas como el diseño logístico de sistemas productivos y el concepto aplicado de ingeniería de ecoeficiencia. Para proceder con la estructuración del proceso de reciclaje y la identificación de los recursos para su funcionamiento; que seguidamente estimó y proyectó la elección de las tecnologías mínimas para las operaciones que lo componen.

Se plasmó la propuesta piloto, con cantidades potenciales y distribución en planta, que cumple con los parámetros de diseño de las unidades de trabajo y sus requisitos mínimos necesarios. Lo anterior, se adoptó a las características del predio Buenos Aires, inmediato al predio del Relleno Sanitario Doña Juana, en la Ciudad. Este procedimiento trazado formuló los lineamientos de la "Unidad Logística de Reciclaje Piloto de RCD para Bogotá D.C.", atendiendo lo determinado en el Plan de Ordenamiento Territorial - POT de la región respecto a su ubicación y razón social [5], como un estándar de gestión ecoeficiente vinculado con el sistema logístico. 
En el estudio se siguieron parámetros delineados a nivel internacional por los países que lideran estos procedimientos y la normatividad propia o correlata, para la propuesta del proceso de aprovechamiento ambientalmente sostenible de los residuos, donde se obtendrán agregados reciclados que cumplan con características verificables, establecidas en las Normas Técnicas Colombianas, que los hacen competitivos en el mercado de la construcción civil.

El modelo obtenido como solución logística, sigue los parámetros trazados a nivel mundial por los países líderes; atendiendo como técnica la estructuración o diseño piloto del sistema provechoso y la caracterización de los recursos básicos de funcionamiento, así como la distribución con el diseño de módulos ecoeficientes.

\section{ESTADO ACTUAL EN LA GESTIÓN DE RCD}

Recorriendo la historia reciente, a finales de los años 70, en Japón se implementó el primer tratamiento eficiente de los RCD, como resultado de la normativa que obliga a la utilización de agregados reciclados de concreto en las nuevas construcciones. Posteriormente, en la década de los 80 entró en vigencia legislación estricta en países como Dinamarca, Rusia, Alemania, Francia, España, Bélgica, Noruega, Holanda y China. Brasil fue el primer país en América del Sur en adoptar tecnologías de reciclaje al instalar una planta de RCD, fundamentada en la Resolución del Congreso Nacional del
Medio Ambiente, Brasil - CONAMA No. 307 del 2002, estableciendo directrices para su gestión. [6]

Por tanto, el reciclaje de éstos es considerado como una de las estrategias para la "Producción Más Limpia", lo que la convierte en uno de los planes de acción de los países menos desarrollados, para alcanzar la sostenibilidad en el sector [3].

Comparando el nivel de tecnificación, se identifica la gran brecha que posee Colombia en relación a los países europeos, ya que por ejemplo, España cuenta actualmente con 120 instalaciones de este reciclaje [7]. Para el País una de las experiencias realizadas es el vertido en sitios de acumulación, presentando escasa alternativa de valorización, reutilización o reciclaje (menos del 5\% son sometidos a estos procesos, que en su mayoría son de tipo artesanal); como también la práctica ilegal en gran parte de los casos [8].

Para el caso de la ciudad de Bogotá D.C., se ha manifestado una innegable falta de organización y control respecto al manejo, que presentó una tasa de crecimiento para el 2012 proyectada promedio de $9,7 \%$ en el sector público y $4,4 \%$ en el sector privado [3]; lo que ha generado impactos socioambientales y económicos negativos, como obstaculización en el tránsito, obstrucción de los sistemas de alcantarillado, aumento en costos de mantenimiento, probabilidades de inundación, disminución en la vida útil de los rellenos sanitarios y alteración en las características eco-sistémicas de los 
humedales y otros cuerpos de agua de la región, entre otras [9].

En la ciudad existen entidades públicas responsables del manejo, que están generando conciencia del aprovechamiento y potencial valorización del material inerte de obras civiles como la Secretaria Distrital de Ambiente - SDA y la Unidad Administrativa Especial de Servicios Públicos - UAESP. La segunda, es la entidad pionera en el control de esta problemática en la que está inmersa la ciudad; así como también, es el ente encargado de vigilar y gestionar la recolección, transporte y disposición final de los RCD producidos y aquellos que están dispuestos en sitios públicos no autorizados para ello. [10] [11].

Actualmente, existen otras entidades particulares que adoptan estrategias para la construcción y/o instalación de infraestructuras que ayudan a la minimización de los problemas generados por la gestión de RCD. Éstas, llamadas "escombreras autorizadas", no poseen la suficiente capacidad para suplir la gran demanda de los generados por el auge del sector. Se presenta además, baja oferta de sitios de disposición final; obedeciendo a factores relacionados con los altos costos de la tierra, ausencia de predios extensos libres que cumplan con los requerimientos legales, ambientales y sociales, exigidos por el Ministerio de Ambiente y Desarrollo Sostenible y atendiendo lo determinado en el Plan de Ordenamiento Territorial - POT, que impiden la localización de los mismos en predios urbanos [8].

El País tiene una legislación estructurada referente al tema de manejo y aprovechamiento de éstos con el fin de garantizar el "Desarrollo Sostenible"; definida según la Comisión Mundial sobre el Medio Ambiente y Desarrollo como: "aquel que satisface las necesidades del presente sin comprometer la capacidad de las futuras generaciones de satisfacer sus propias necesidades"; con sus tres componentes: "Medio ambiente, sociedad y economía" [12].

El Estado colombiano integró en su función legislativa los preceptos registrados en las normas descritas en la Tabla No. 1, definida con información del "Anteproyecto para la Gestión Integral de RC\&D en el Distrito Capital. COAMBIENTE. 2009" [8].

Tabla 1. Normatividad Vigente en Colombia respecto al manejo de Residuos

\begin{tabular}{|c|l|}
\hline \multicolumn{2}{|c|}{ NORMATIVIDAD VIGENTE PARA EL MANEJO DE RESIDUOS } \\
\hline NOMBRE & \multicolumn{1}{c|}{ DESCRIPCIÓN } \\
\hline $\begin{array}{c}\text { Constitución Política } \\
\text { de Colombia 1991 }\end{array}$ & $\begin{array}{l}\text { El Título II, Capítulo III, Artículo 80, dispone "El Estado planificará el manejo } \\
\text { y aprovechamiento de los recursos naturales, para garantizar su desarrollo } \\
\text { sostenible, su conservación, restauración o sustitución". }\end{array}$ \\
\hline
\end{tabular}




\begin{tabular}{|c|c|}
\hline Decreto 2811 de 1974 & $\begin{array}{l}\text { "Código Nacional de Recursos Naturales Renovables y de Protección al Medio } \\
\text { Ambiente", que regula el manejo de residuos, basuras, desechos y desperdicios. } \\
\text { Relacionando el tema en estudio dispone: "Reintegrar al proceso natural y } \\
\text { económico los desperdicios sólidos, líquidos y gaseosos, provenientes de } \\
\text { industrias, actividades domésticas o de núcleos humanos en general" y } \\
\text { "Perfeccionar y desarrollar nuevos métodos para el tratamiento, recolección, } \\
\text { depósito, y disposición final de los residuos sólidos, líquidos o gaseosos no } \\
\text { susceptibles de nueva utilización" }\end{array}$ \\
\hline \multirow[b]{2}{*}{ Ley 99 de 1993} & $\begin{array}{l}\text { Creó el Ministerio del Medio de Ambiente y reordenó el sector público encargado } \\
\text { de la gestión y conservación del Medio Ambiente y de los Recursos Naturales } \\
\text { Renovables. Organizó el Sistema Nacional Ambiental, SINA. }\end{array}$ \\
\hline & $\begin{array}{l}\text { El Artículo } 2 \text { regula: "las condiciones generales para el saneamiento del medio } \\
\text { ambiente, y el uso, manejo, aprovechamiento, conservación, restauración y } \\
\text { recuperación de los recursos naturales, a fin de impedir, reprimir, eliminar o } \\
\text { mitigar el impacto de actividades contaminantes, deteriorantes o destructivas del } \\
\text { entorno o del patrimonio natural". }\end{array}$ \\
\hline $\begin{array}{c}\text { Decreto } 605 \text { de marzo } \\
\text { de } 1996\end{array}$ & $\begin{array}{l}\text { Reglamenta la Ley } 142 \text { de } 1994 \text { en relación con la prestación del servicio público } \\
\text { domiciliario de aseo. Asigna la responsabilidad a las entidades locales en la } \\
\text { gestión de los residuos urbanos y escombros procedentes de la construcción. El } \\
\text { Artículo } 46 \text { determina que "Es responsabilidad de los productores de escombros } \\
\text { su recolección, transporte y disposición en las escombreras. Las entidades } \\
\text { prestadoras del servicio de aseo en la zona son responsables de coordinar } \\
\text { estas actividades. La recolección, transporte y disposición de escombros deberá } \\
\text { efectuarse en forma separada del resto de residuos sólidos". Además el Artículo } \\
79 \text { determina que "su disposición debe realizarse en escombreras previamente } \\
\text { definidas por el municipio de conformidad a las disposiciones ambientales } \\
\text { vigentes" }\end{array}$ \\
\hline Ley 142 de 1994 & $\begin{array}{l}\text { "Por la cual se establece el régimen de los servicios públicos y se dictan otras } \\
\text { disposiciones" cita en el Artículo } 14 \text { numeral 14.24, que: "el tratamiento, } \\
\text { aprovechamiento y disposición final de los residuos sólidos son actividades } \\
\text { complementarias del servicio público domiciliario de aseo". }\end{array}$ \\
\hline $\begin{array}{c}\text { Resolución } 541 \\
\text { Diciembre } 14 \text { de } 1994\end{array}$ & $\begin{array}{l}\text { Regula "el cargue, descargue, transporte almacenamiento y disposición final de } \\
\text { escombros, materiales, elementos concretos y agregados sueltos de construcción, } \\
\text { de demolición y capa orgánica, suelo y subsuelo de excavación". Define parámetros } \\
\text { ambientales para la construcción y operación de escombreras utilizadas para la } \\
\text { disposición final de estos materiales. }\end{array}$ \\
\hline
\end{tabular}




\begin{tabular}{|c|l|}
\hline $\begin{array}{c}\text { Decreto Distrital } 357 \\
\text { de } 1997\end{array}$ & $\begin{array}{l}\text { "Por el cual se regula el manejo, transporte, y disposición final de escombros y } \\
\text { materiales de construcción en el Distrito Capital". }\end{array}$ \\
\hline $\begin{array}{c}\text { Decreto Nacional 1713 } \\
\text { de } 2002 \text { y Decreto } 838 \\
\text { de } 2005\end{array}$ & $\begin{array}{l}\text { En su Artículo 44, menciona que: "la recolección de escombros es responsabilidad } \\
\text { escombreras autorizadas". "El Municipio y las personas prestadoras del servicio } \\
\text { de aseo son responsables de coordinar estas actividades en el marco de los } \\
\text { programas establecidos para el desarrollo respectivo de Plan de Gestión Integral } \\
\text { de Residuos sólidos -PGIRS-". }\end{array}$ \\
\hline $\begin{array}{c}\text { Decreto Distrital } 312 \\
\text { de } 2006 \text { y Decreto } \\
\text { Distrital } 620 \text { de } 2007\end{array}$ & $\begin{array}{l}\text { Adopta el "Plan Maestro Integral de Residuos Sólidos". Describe el Programa de } \\
\text { recolección, disposición final y tratamiento de residuos hospitalarios, peligrosos, } \\
\text { escombros y lodos. Además propone la instalación de un predio distrital para el }\end{array}$ \\
\hline
\end{tabular}

Fuente: Anteproyecto para la gestión Integral de RC\&D en el Distrito Capital. COAMBIENTE. 2009.

\section{TENDENCIAS Y HERRAMIENTAS DE APOYO}

Para el diseño de la "Unidad Piloto Productiva" es importante tener definidos varios conceptos que apoyan la formulación de la propuesta, como son: Los sistemas productivos logísticos para el procesamiento de materias primas e insumos; así como también, el concepto de ingeniería aplicada basado en la Ecoeficiencia y su gestión.

\subsection{Diseño de Sistemas Productivos Logísticos}

Un Sistema Productivo Logístico, es el conjunto de objetos que se relacionan entre sí para procesar materias primas e insumos, convirtiéndolos en el producto definido por el objetivo del sistema.

Para el diseño de esa unidad productiva, fue importante tomar decisiones estratégicas como [13]:
- Definir la localización de la Planta o Unidad, lugar físico donde se ubicará la industria teniendo en cuenta necesidades de producción, almacenaje y distribución. Tanto como, atendiendo lo ordenado en el POT.

- Definir la capacidad productiva de la planta, lo que significa dimensionar las instalaciones para el volumen de manufactura definido.

- Seleccionar el proceso de producción y la tecnología, elegidas según las características del producto a fabricar y la tecnología disponible.

- Seleccionar la infraestructura de servicios, como conjunto de elementos que permiten el funcionamiento correcto y sucesivo de las instalaciones, como lo son el mantenimiento, el control de calidad, entre otras. 
- Definir el perfil y la cantidad de personal que requiere la empresa, delimitando el nivel de calificación y volumen del personal necesario.

\subsection{Concepto de Ecoeficiencia}

La aplicabilidad de este concepto en los sistemas de ingeniería se refleja en el diseño de la "Unidad", conjugado con el concepto de Ecoeficiencia; definida según la World Business Council for Sustainable Development (WBCSD) como el "Proporcionar bienes y servicios a un precio competitivo, para satisfacer las necesidades humanas y la calidad de vida, al tiempo que se reduzca progresivamente el impacto ambiental y la intensidad de la utilización de recursos a lo largo del ciclo de vida, hasta un nivel compatible con la capacidad de carga estimada del planeta" [14].

Este modelo ecoeficiente en los procesos aumenta la competitividad ya que permite reducir: El manejo inadecuado de los recursos, el volumen de los residuos generados, el consumo de energía y las emisiones contaminantes. Lo anterior favoreciendo las relaciones con la administración. También, al estar relacionado directamente con la sostenibilidad, optimiza tres objetivos principalmente: Crecimiento económico, equidad social y valor ecológico. [14]

\section{METODOLOGÍA}

El diagnóstico permitió la cuantificación, caracterización y análisis de los RCD de la ciudad, en especial los generados en las localidades de Tunjuelito, Ciudad Bolívar, Bosa, Kennedy y Usme; que constituyen el área de influencia del predio seleccionado. La cuantificación se obtuvo con datos suministrados por la SDA y la UAESP [15]. La caracterización se realizó con muestras tomadas de los agregados procesados por los "Molineros Artesanales" del área y datos de estudios previos, para su posterior confrontación y análisis.

Se procedió con la estructuración o diseño del sistema productivo logístico y la identificación de los recursos básicos para su funcionamiento; lo que consecuentemente, llevó a la evaluación y elección de las tecnologías necesarias para cada una de las operaciones que lo componen, teniendo en cuenta diferentes aspectos como tipo, capacidad y características de cada una de las máquinas y equipos. Seguido a esto, se realizó una distribución en planta potencial que cumple con los parámetros de diseño de los módulos de trabajo, adoptando las particularidades del predio pre-destinado tanto como lo estipulado en las consideraciones del POT. Finalmente, se concibió un modelo de gestión, que en conjunto con el sistema logístico generará valor agregado a partir de la reutilización y reciclaje de los producidos; cumpliendo con características competitivas en el mercado de la construcción civil de la región.

Este modelo integra el cumplimiento de la Legislación Colombiana (Descrita en la Tabla 1) y la referencia de normativas estándares internacionales, como son: a) Los requisitos de un sistema de gestión de calidad; b) Los requisitos para un sistema de gestión ambiental, la definición de las políticas, la identificación, evaluación y mitigación de impactos ambientales; c) El asegurar las condiciones básicas de salud ocupacional y seguridad in- 
dustrial; d) Pautas de responsabilidad social de la administración municipal, mitigantes de las diferencias en materia cultural, ambiental, económicas y legal; e) El definir un análisis y gestión de riesgos, eventualidades y contingencias. Lo anterior, apoyados en las normas colombianas: NTC ISO 9001: 2008 [16]; NTC ISO 14001:2004 [17]; NTC OSHAS ISO 18001:2007 [18]; NTC ISO 26000: 2010 [19] y NTC ISO 31000:2011 [20].

En general, se representa con la propuesta un cambio de mentalidad respecto a estos RCD, ya que se convierten en material valorizable o con valor agregado para el uso en bien de la comunidad y su entorno.

\section{ESTUDIO DE CASO - PROPUESTA MODELO DE GESTIÓN ECOEFICIENTE}

Constitución Política de Colombia, Título II, Capítulo III, Artículo 80: "El Estado planificará el manejo y aprovechamiento de los recursos naturales, para garantizar su desarrollo sostenible, su conservación, restauración o sustitución".

La ubicación potencial selecta fue el predio denominado Buenos Aires [6] para el cual, según el Informe de Gestión de la Secretaría Distrital del Hábitat, ya fue gestionado el certificado de viabilidad ambiental como escombrera distrital; este centro de aprovechamiento, es definido como un sitio en donde se podrán transformar y almacenar de manera temporal los RCD, considerando las medidas ambientales que prevengan y/o mitiguen los impactos generados que requerirán aprobación de las autoridades ambientales y sanitarias [12].

En la Figura 1 se presenta la localización del predio, planteado como sitio de aprovechamiento y disposición final para el distrito por la UAESP [3].

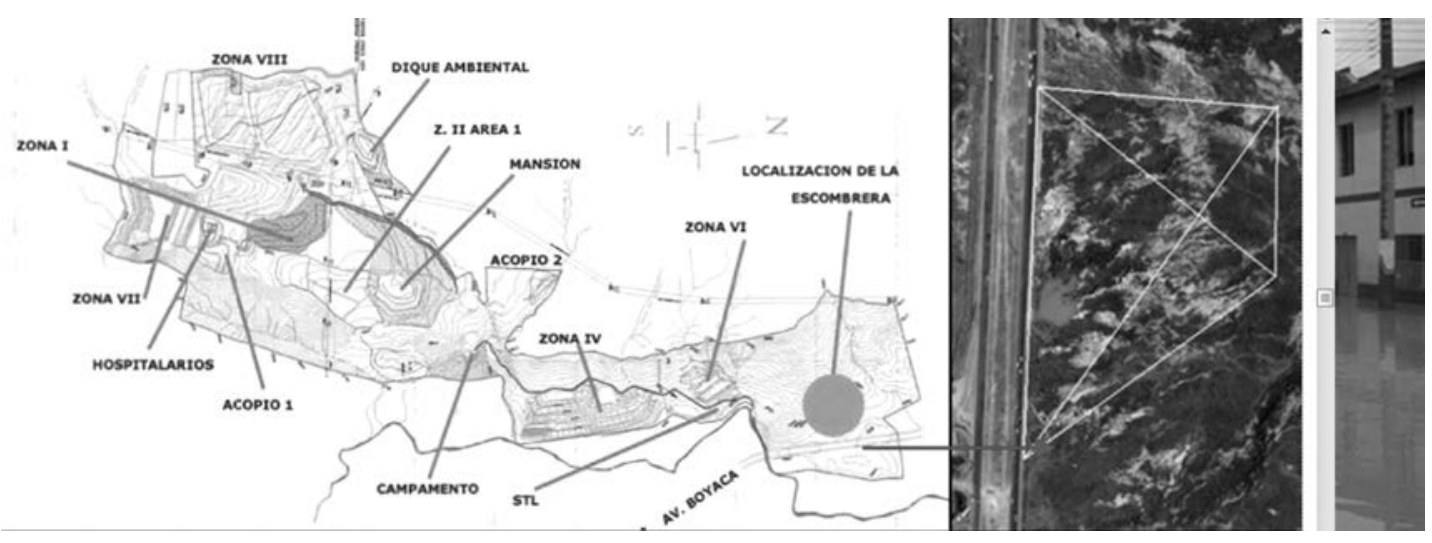

Figura 1. Localización del Predio Buenos Aires. Planteado como sitio de aprovechamiento y disposición final de RCD para el Distrito por la UAESP.

Fuente: $\cup A E S P$. Anexos 1 y 5, Diagnóstico del manejo integral de escombros en Bogotá. 2009. 
Según los términos de referencia para la disposición final de RCD y el POT [5], los procesos que involucran el almacenamiento y manejo deben localizarse en áreas donde el paisaje esté degradado, para que estos materiales contribuyan a su restauración. Siendo así y cumpliendo con estas características, el predio se localiza al costado de la Autopista al Llano (Avenida Boyacá), al margen derecho de la entrada del "Relleno Sanitario Doña Juana", con un área aproximada de 167 hectáreas [3]. El lugar de instalación de la Unidad es fundamental para la aplicación del programa de reciclaje, considerándose importante que se encuentre próxima a la fuentes generadoras del residuo y a los lugares de aplicación del agregado reciclado, es decir dentro del contexto urbano [6]. La ubicación de la planta es fija, lo que permite maquinaria de grandes dimensiones para la capacidad prevista. Estimando la cantidad generada en el radio de influencia, como también la producción de material reciclado [21].

Utilizando la metodología de un procedimiento general de logística en reversa, defi-
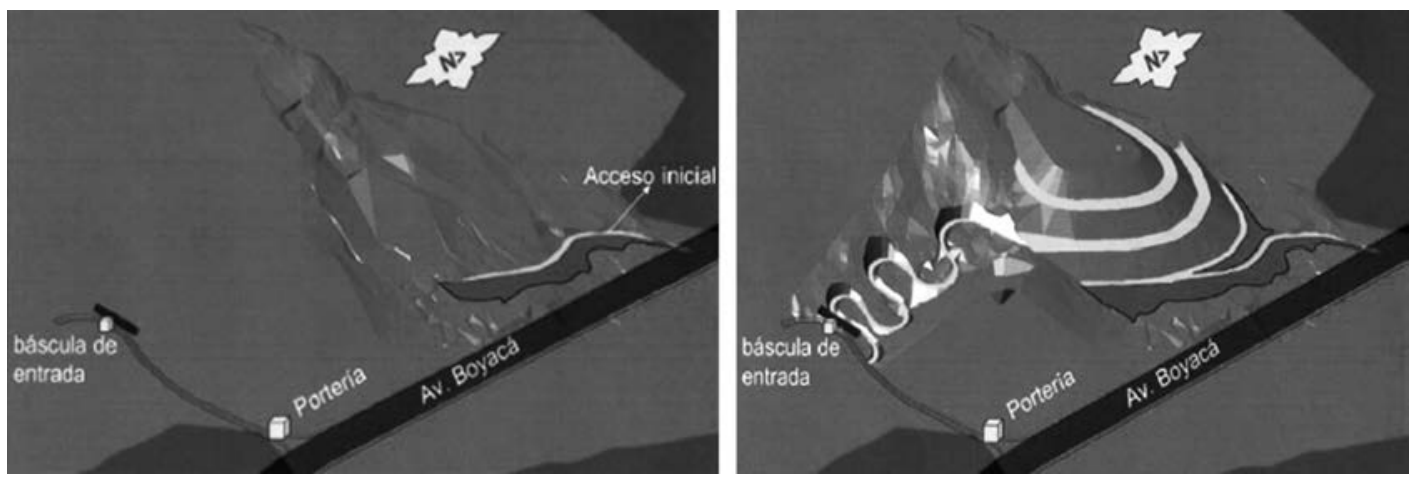

Figura 2. Vías de acceso Predio Buenos Aires

Fuente: UAESP. Anexo 1, Diagnóstico del manejo integral de escombros en Bogotá. 2009. nida como la gestión del flujo de productos; destinados al reprocesamiento, reciclaje, reutilización o destrucción, mediante la aplicación de actividades de recolección y recuperación ecológica sostenida [22], se propone el análisis de diferentes decisiones estratégicas para un adecuado diseño del sistema productivo; como son: Caracterización del producto a procesar, selección y diseño de proceso, integración de ecnologías, volumen de producción, localización, distribución en planta, calidad del proceso, personal, entre otros [23].

Según un análisis de factibilidad técnica para la localización de una escombrera en este sitio, realizado por la UAESP y en concordancia con los términos de referencias, es necesario realizar unas obras de adecuación y preparación del sitio, para lo cual se han propuesto dos vías de acceso. La Figura 2 muestra las vías de acceso posibles al predio. Una desde la Avenida Boyacá y la otra a un costado de la propiedad, donde se encuentra actualmente la báscula de entrada del Relleno.

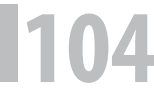

Diciembre de 2013 
Se realizó un diagnóstico de la situación identificando y cuantificando las variables, principalmente el volumen a tratar, para lo cual se utilizó la proyección de la cantidad según fuentes de generación para el año 2012, en la ciudad, como se observa en la Tabla No. 2.

Tabla 2. Volumen de escombros generados en la ciudad de Bogotá, 2012, según proyecciones realizadas con datos de la UAESP

\begin{tabular}{|c|c|c|}
\hline GENERADOR & CANTIDAD & UNIDADES \\
\hline Sector Privado & 8.083 .159 & $\mathrm{~m}^{3}$ \\
\hline Sector Público & 5.944 .285 & $\mathrm{~m}^{3}$ \\
\hline Clandestinos* & 202.961 & $\mathrm{~m}^{3}$ \\
\hline Total & $\mathbf{1 4 . 2 3 0 . 4 0 5}$ & $\mathrm{m}^{3}$ \\
\hline
\end{tabular}

Fuente: $\cup A E S P$. Diagnóstico del manejo integral de escombros en

Bogotá. 2009.

Secretaria Distrital de Hábitat. 2008.

Con una cantidad anual total de éstos constituyen la materia prima de un 14.230.405 $\mathrm{m}^{3}$, se obtiene un volumen proceso de transformación, fue necesario promedio de $38.987 \mathrm{~m}^{3} / \mathrm{dí}$. Una vez es- realizar una caracterización previa para tudiado y teniendo en cuenta el área de conocer su constitución y heterogeneiinfluencia de la zona y los puntos de ge- dad. En la Tabla No. 3 se observa la comneración, se trazó como objetivo inicial la posición resultante de muestras en Bogestión de una cantidad diaria de 13.412 gotá, con una distribución porcentual de $\mathrm{m}^{3} / \mathrm{dí}$, que representan un $34,4 \%$ de los cada uno de ellos [24]. $\mathrm{RCD}$ generados en la ciudad. Debido a que 
Tabla 3. Composición de los RCD en Bogotá

\begin{tabular}{|c|c|c|}
\hline COMPOSICIÓN & TONELADAS & PORCENTAJE \\
\hline Residuos Orgánicos & 0,115 & $0,006 \%$ \\
\hline Otros Escombros & 55,218 & $3,011 \%$ \\
\hline Vidrio & 37,062 & $2,021 \%$ \\
\hline Tierra & 345,930 & $18,864 \%$ \\
\hline Textiles & 16,885 & $0,921 \%$ \\
\hline Plásticos & 20,653 & $1,126 \%$ \\
\hline Papel & 1,521 & $0,083 \%$ \\
\hline Mármol & 12,152 & $0,663 \%$ \\
\hline Madera & 45,151 & $2,460 \%$ \\
\hline Lámina de yeso & 19,364 & $1,056 \%$ \\
\hline Ladrillo & 338,538 & $18,461 \%$ \\
\hline Icopor & 7,433 & $0,405 \%$ \\
\hline COMPOSICIÓN & TONELADAS & PORCENTAJE \\
\hline Hierro y Bronce & 0,204 & $0,011 \%$ \\
\hline Guadua & 3,813 & $0,208 \%$ \\
\hline Gres & 78,505 & $4,281 \%$ \\
\hline Granito & 45,165 & $2,463 \%$ \\
\hline Concreto & 499,051 & $27,214 \%$ \\
\hline Cobre & 2,570 & $0,140 \%$ \\
\hline Caucho & 10,643 & $0,580 \%$ \\
\hline Cartón & 0,397 & $0,022 \%$ \\
\hline Cerámica & 167,621 & $9,141 \%$ \\
\hline Asfalto & 21,874 & $1,193 \%$ \\
\hline Asbesto & 76,570 & $4,175 \%$ \\
\hline Arena & 23,751 & $1,295 \%$ \\
\hline Agregados & 1,505 & $0,082 \%$ \\
\hline Acero & 2,103 & $0,115 \%$ \\
\hline Totales & 1833,794 & $100,000 \%$ \\
\hline
\end{tabular}

Fuente: A. Chávez, 2013 [24]

A partir de la clasificación de los RCD en Brasil, según la normativa CONAMA, como se observa en la Tabla No. 4 [25] y extrapolando para la ciudad de Bogotá, éstos fueron considerados como Clase $A$; los cuales pue- den ser reciclados y utilizados como agregados en el sector de la construcción, es decir los potenciales a ser procesados por la Unidad a diseñar. Con la misma analogía, respecto a los residuos clasificados como 
Clase $B$, éstos serán enviados a la planta de reciclaje específica ya que representan cantidades de materiales como papel, plástico, metal, vidrio, madera, etc.

Tabla 4. Clasificación de los Residuosde Construcción y Demolición en Brasil, CONAMA

\begin{tabular}{|l|c|}
\hline CLASE A & $\begin{array}{c}\text { Residuos reutilizables o reciclables } \\
\text { como agregado para } \\
\text { la construcción }\end{array}$ \\
\hline CLASE B & $\begin{array}{c}\text { Residuos reciclables para otrosusos } \\
\text { Residuos de los que no se han } \\
\text { desarrollado tecnologías o } \\
\text { aplicaciones, económicamente } \\
\text { viables que permitan su reciclaje } \\
\text { y recuperación }\end{array}$ \\
\hline CLASE D & $\begin{array}{c}\text { Desechos peligrosos } \\
\text { desde el proceso de construcción }\end{array}$ \\
\hline
\end{tabular}

Fuente: Implementação de usina para reciclagem de resíduos da construção civil (RCC) como ação para o desenvolvimento sustentável - estudo de caso. 2008.

Al analizar la composición, se observa que la mayor parte de éstos son reciclables. En la Tabla No. 3 refleja que un $39 \%$ del material no es objetivo para la Unidad (Valores sombreados en la Tabla), lo que reduce el volumen a tratar a $8.181 \mathrm{~m}^{3}$ /día. La cifra anterior, formula la cuantificación del problema a transformar como se muestra en la siguiente ecuación.

$$
C_{R C D}:\left(C_{S P R}+C_{S P}+P C_{C}\right) A I \%-M N R \%
$$

Donde:

$C_{\mathrm{RCD}}$ : Cantidad de residuos a reciclar por día.

$\mathrm{C}_{\mathrm{SPR}}$ : Cantidad de residuos generados por el sector privado.

$C_{\mathrm{SP}}$ : Cantidad de residuos generados por el sector público.

$\mathrm{PC}_{\mathrm{c}}$ : Cantidad promedio de residuos clandestinos.

Al\%: Porcentaje de residuos generado en el área de influencia del predio.

MNR\%: Porcentaje promedio de material no reciclable contenido en los escombros.

El proyecto propuesto se presenta en varias etapas o fases de implementación para lograr la cobertura total. La Tabla No. 5 presenta la capacidad de la planta según Fase de construcción y operación.

Se debe contar con la instalación de 2 Plantas Móviles con sus respectivas Máquinas 1- 2 o 3 lo que permitirá presentar una Capacidad Máxima y una Capacidad Utilizada.

Desde la Fase I se propuso el diseño de un proceso de separación, clasificación, triturado y cribado que permita obtener los materiales valorizables, basado en una línea de producción que describe el conjunto de las operaciones. La Figura No. 3 presenta el diagrama de flujo del proceso de reciclaje de RCD [26]- [31]. 
Tabla 5. Capacidad de la planta según fase de construcción y operación

\begin{tabular}{|c|c|c|c|c|c|}
\hline & FASE I & FASE II & \multicolumn{3}{|c|}{ FASE III } \\
\hline PLANTA MÓVIL 1 & MÁQUINA 1 & MÁQUINA 1 & MÁQUINA 1 & MÁQUINA 2 & MÁQUINA 3 \\
\hline CAPACIDAD MÁXIMA & $450 \mathrm{t} / \mathrm{h}$ & $450 \mathrm{t} / \mathrm{h}$ & $450 \mathrm{t} / \mathrm{h}$ & $450 \mathrm{t} / \mathrm{h}$ & $450 \mathrm{t} / \mathrm{h}$ \\
\hline CAPACIDAD UTILIZADA & $33,3 \%$ & $88,8 \%$ & $88,8 \%$ & $88,8 \%$ & $49,5 \%$ \\
\hline PLANTA MÓVIL 2 & MÁQUINA 1 & MÁQUINA 1 & MÁQUINA 1 & MÁQUINA 2 & MÁQUINA 2 \\
\hline CAPACIDAD MÁXIMA & $400 \mathrm{t} / \mathrm{h}$ & $400 \mathrm{t} / \mathrm{h}$ & $400 \mathrm{t} / \mathrm{h}$ & $400 \mathrm{t} / \mathrm{h}$ & $400 \mathrm{t} / \mathrm{h}$ \\
\hline CAPACIDAD UTILIZADA & $37 \%$ & $100 \%$ & $100 \%$ & $100 \%$ & $55,7 \%$ \\
\hline CANTIDAD DE ESCOMBROS A TRATAR & $150 \mathrm{~m}^{3} / \mathrm{h}$ & $400 \mathrm{~m}^{3} / \mathrm{h}$ & \multicolumn{3}{|c|}{$1.022 \mathrm{~m}^{3} / \mathrm{h}$} \\
\hline CANTIDAD DE ESCOMBROS A TRATAR & $1.200 \mathrm{~m}^{3} / \mathrm{d}$ ía & $3.200 \mathrm{~m}^{3} / \mathrm{dí}$ & \multicolumn{3}{|c|}{$8.181 \mathrm{~m}^{3} / \mathrm{d}$ ía } \\
\hline
\end{tabular}

\subsection{Línea de Producción}

- Recepción del Material. Los vehículos que ingresan son sometidos a los procesos de pesaje y basculación, mediante un programa informático se conoce el volumen de material que ingresa.

- Control. Tras el pesaje, el material de entrada es inspeccionado visualmente, observando que no ingresen elementos que contaminen el proceso u ocupen un lugar no previsto.

- Clasificación primaria. Luego de la descarga se procede a la separación, teniendo en cuenta las características propias de composición. Puede realizarse manualmente o combinada con medios mecánicos; aquí se obtienen materiales como metales, maderas, plásticos, compuestos orgánicos, elementos visibles, etc. Los separados en esta operación son depositados en acopios o contenedores adecuados para cada tipo, donde son almacenados para ser entregados a la empresa de gestión autorizada.

- Clasificación por tamaños o granulométrica. Se realiza con equipos mecánicos de tamizado, consistentes en separar las partículas grandes de las pequeñas por medio del movimiento a través de unas mallas o láminas perforadas. Este proceso se complementa con el transporte de material de diferente granulometría a través de las bandas mecánicas.

- Siguiendo la secuencia del proceso (Cribado) el material de entrada es cargado a la tolva de alimentación y de aquí a la cinta dosificadora que conduce al trómel de clasificación donde se dividen en dos fracciones: 0-60 mm y > $60 \mathrm{~mm}$. Estas cintas transportadoras están diseñadas especialmente para este tipo de material; presenta un ancho útil de 1,000 $\mathrm{mm}$ y un carenado especial para reducir el efecto del viento con el fin de prevenir el arrastre de material ${ }^{[26]}$. La fracción de material > $60 \mathrm{~mm}$ y < $250 \mathrm{~mm}$ será sometida a un proceso de separación manual con las mismas características de la clasificación primaria, descrita anteriormente. Inmediatamente después de este proceso, es conducido a la trituradora primaria que lo muele y conduce a una criba vibratoria (Cribado) donde nuevamente se clasifica en dos fracciones, < $35 \mathrm{~mm}$ y $>35 \mathrm{~mm}$. 
UNIDAD LOGÍSTICA DE RECUPERACIÓN DE RESIDUOS DE CONSTRUCCIÓN Y DEMOLICIÓN: ESTUDIO DE CASO BOGOTÁ D.C.

\begin{tabular}{|l|c|}
\hline \multicolumn{1}{|c|}{ OPERACIONES } & \multirow{2}{*}{ PERSONAL } \\
\cline { 1 - 1 } Ingreso de Material & 1 \\
\hline Pesaje de Material & 6 \\
\hline Separación Manual & 1 \\
\cline { 1 - 1 } Transporte a Tolva & \\
\cline { 1 - 1 } Trituracion Primaria & \multirow{2}{*}{0} \\
\cline { 1 - 1 } Cribado de Material & \\
\cline { 1 - 1 } Separación por Aire & \\
\hline Separación Magnética & \\
\hline Trituración Secundaria & 1 \\
\hline Cribado de Material & 1 \\
\hline Director de Planta & 6 \\
\hline Secretaria & 2 \\
\hline Almacén & 15 \\
\hline Laboratorio & 2 \\
\hline Conductores & 3 \\
\hline Depto. Financiero & 1 \\
\hline Depto. Comercial & 1 \\
\hline Depto. Calidad & 40 \\
\hline Depto, HSEQ & \\
\hline TOTAL & \\
\hline
\end{tabular}
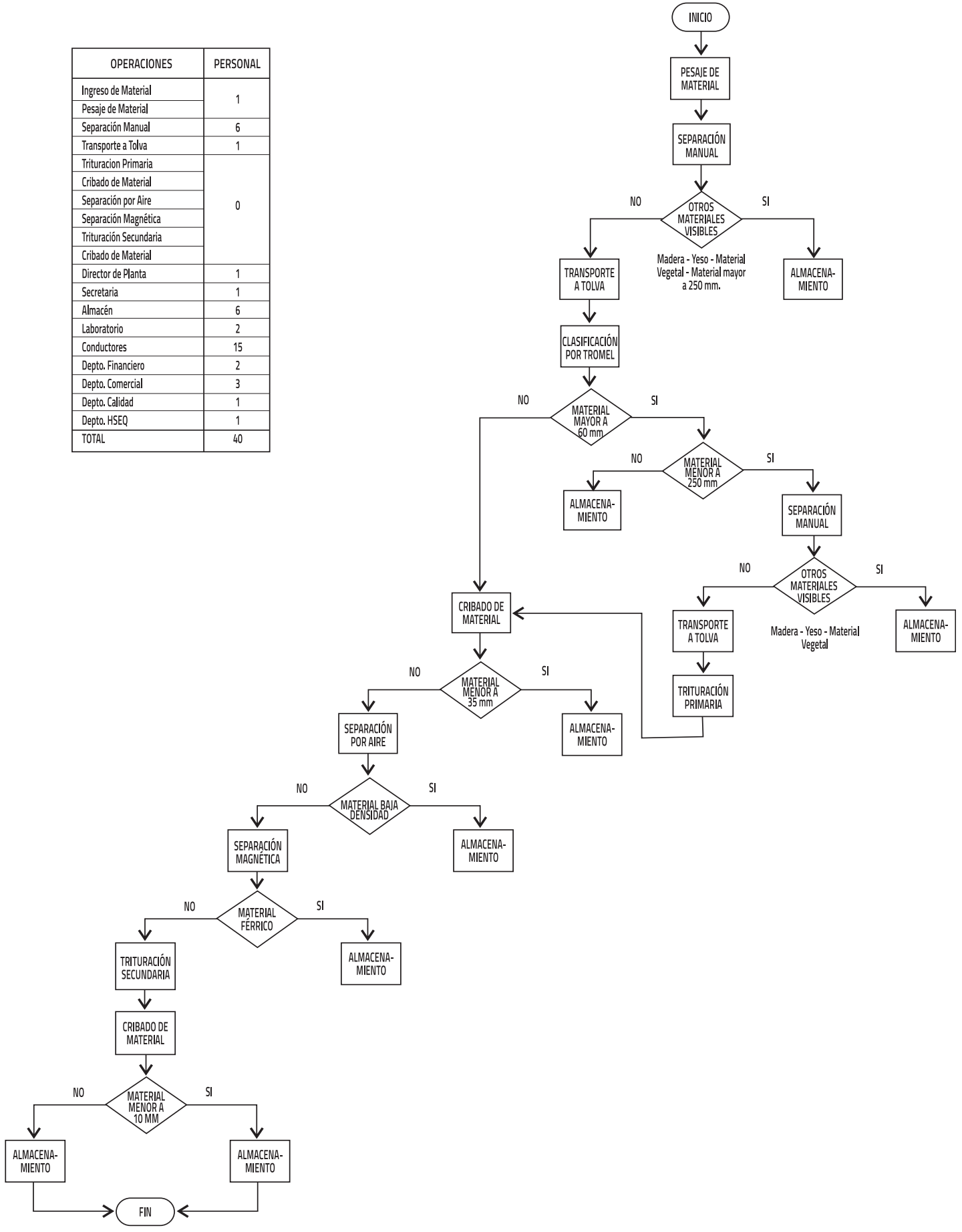

Figura 3. Diagrama de flujo del proceso de reciclaje de escombros

Fuente: Los autores. 2012. 
- Operaciones de trituración primaria. Donde se reduce el tamaño, existe la trituración primaria y secundaria según sea el tamaño alimentado y la granulometría del producto requerido. Dentro del proceso se implementará inicialmente la primaria, en donde las partículas que tienen una granulometría > $35 \mathrm{~mm}$ luego de ser trituradas, siguen con las operaciones de separación magnética y neumática.

- Clasificación neumática. Después de la trituración primaria se realiza una separación neumática utilizada para retirar fragmentos de los elementos de baja densidad, como son los papeles y plásticos, que aún están contenidos produciendo contaminación.

- Separación magnética. Al mismo tiempo, se retiran los elementos metálicos valorizables que obstaculizan la fase siguiente del proceso, utilizando un electroimán.

- Operaciones de trituración secundaria. Donde el material > 35 mm es sometido de nuevo a un proceso de pulverización para obtener fracciones más finas, que finalizan el proceso al ser cribadas nuevamente, obteniendo como producto final agregados reciclados con granulometrías < $10 \mathrm{~mm}$ que comercialmente se conocen como agregados finos.
Una vez estructurado el proceso, se procede con la elección de la tecnología a utilizar en cada una de las operaciones, analizando el tipo, capacidad, características y dimensiones de cada una de las máquinas y equipos necesarios:

\subsection{Para la Trituración}

- Las trituradoras de rodillo o cono, de flujo horizontal, reduce el tamaño de la partícula de acuerdo con la proximidad de la base del alimentador de placas que le suministra material.

- Las trituradoras de mandíbulas, pueden ser de flujo horizontal o vertical. Son de construcción muy resistente, con entrada para elementos voluminosos y fiabilidad de funcionamiento.

- Las trituradoras de impacto, que disponen de un rotor provisto de barras que lanzan el material contra las paredes internas, revestidas con placas de acero anti abrasivo, logrando tamaños menores en relación muy alta a lo alimentado [32].

Analizadas las características de cada una de las trituradoras, según la revisión bibliográfica, se presenta un cuadro comparativo teniendo en cuenta diferentes propiedades [33], como se observa en la Tabla No. 6. 
Tabla 6. Comparación proceso tecnológico de trituración

\begin{tabular}{|l|c|c|c|}
\hline PROPIEDADES & CONOS & MANDÍBULAS & IMPACTO \\
\hline Capacidad & Media & Alta & Bajo \\
\hline Costos de Producción & Medio & Bajo & Alto \\
\hline Desgaste & Bajo & Bajo & Alto \\
\hline Calidad del Árido & Medio & Baja & Alta \\
\hline Contenido de Finos & Medio & Bajo & Alto \\
\hline Consumo de Energía & Medio & Bajo & Alto \\
\hline
\end{tabular}

Fuente: Plantas Recicladoras de RCD. Jorge Mosquete [33]

Observando los datos anteriores y fundamentando la decisión en las unidades implementadas a nivel mundial, la trituradora más apropiada para la etapa primaria es la de mandíbulas y para la secundaria, por la calidad del árido, se eligió la de Impacto.

\subsection{Para la clasificación neumática}

Ésta se elegirá según la disponibilidad en el mercado.

- De aspiración vertical. Consiste en un alimentador que almacena el material cribado sobre un depósito cilíndrico unido a un ventilador, el cual crea una depresión que aspira los ligeros por la parte superior.

- Criba neumática. Consiste en una bandeja inclinada con un tamiz a través del cual se introduce aire desde la parte inferior. El material ligero es expulsado hacia un lateral mientras el pesado continúa su avance.

- Túnel de viento de doble efecto. Mediante una corriente de aire los materiales ligeros son desplazados a la salida de un transportador [32].
Haciendo una evaluación y elección de la maquinaria se procedió a la aproximación de un Layout que contempla la Fase I, abarcando inicialmente una cantidad de RCD del $15 \%$ aproximado de la cantidad total objetivo por día de la Unidad $\left(1,200 \mathrm{~m}^{3}\right)$, como se observa en la Figura No. 4; la cual presenta la distribución de planta inicial, teniendo en cuenta la secuencia del proceso.

Para el diseño dentro de la planta deben existir áreas de circulación, carga y descarga de camiones y contenedores diseñados de forma que permitan la revisión y primera valoración de los RCD. El almacenamiento inicial se debe realizar de forma diferenciada, según el tipo (Concreto, cerámicos, asfaltos, etc.), para garantizar la calidad del producto obtenido [34].

Para el diseño de estas zonas se tuvieron en cuenta tres aspectos fundamentales:

Número de muelles

Espacios de maniobra de vehículos

Ubicación de las volquetas. 


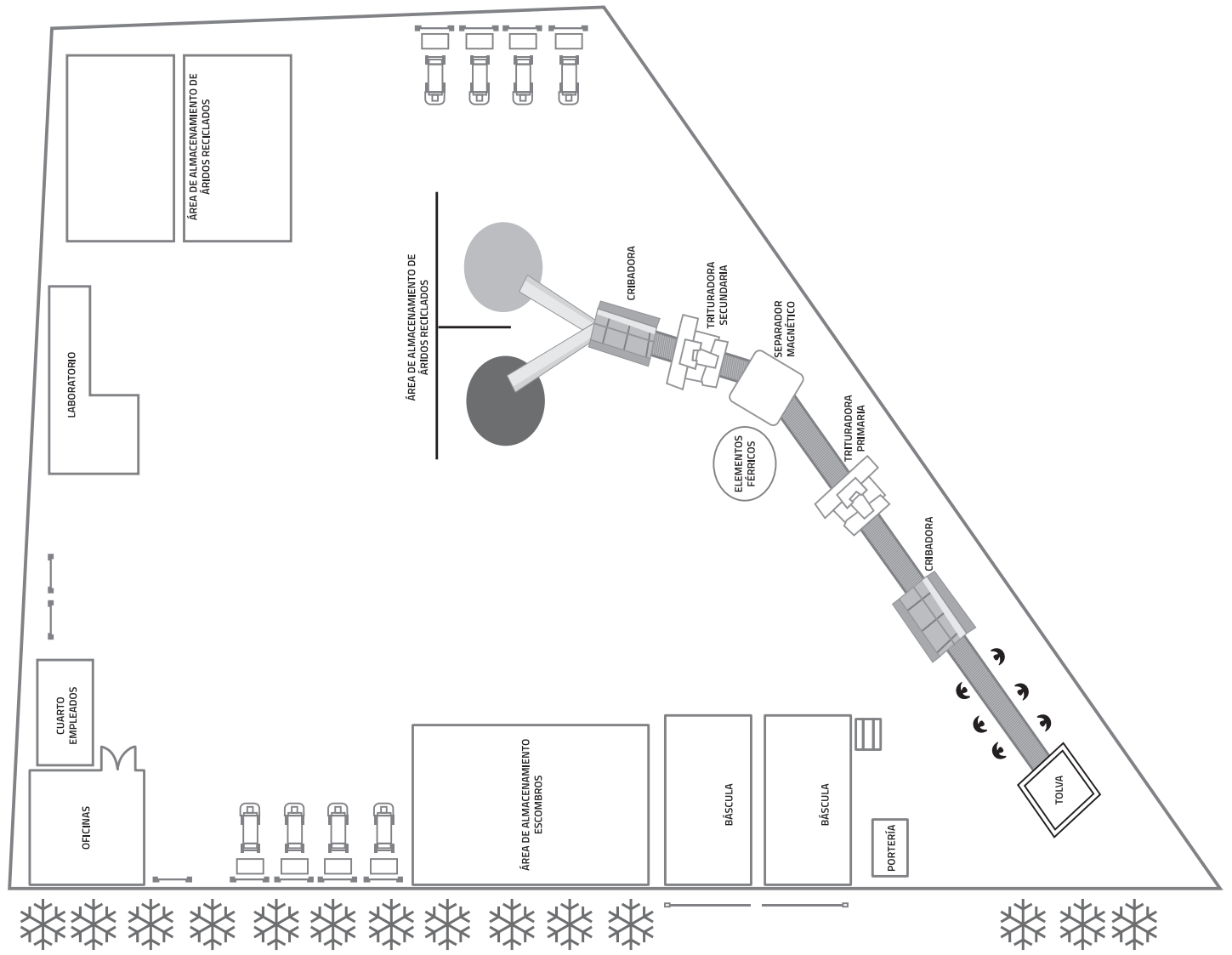

Figura 4. Distribución de planta inicial teniendo en cuenta la secuencia del proceso.

Fuente: Los autores. 2012.

En el presente caso, se eligió el diseño de muelles con descarga posterior, ya que además de minimizar el área de uso, disminuirá el riesgo de accidentes en la zona.

Por otro lado, la Unidad propuesta en la Tabla No. 6, una vez evaluada, para su funcionamiento debe prever condiciones de control de ruido, olor, generación de partículas, descargas líquidas y control de vectores de transmisión, tanto como cumplir con las siguientes características [28]:
- Se propuso la selección de una estructura tecnológica modular en tres fases para facilitar su construcción y operación, la cual consta de dos plantas móviles de trituración y una criba móvil, tal como se describe en la Tabla No. 5 [35].

- La móvil primaria está constituida por una trituradora de mandíbulas, con capacidad máxima de 450 t/h (En la FASE I tendrá una capacidad utilizada del $33,3 \%)$. 
- La segunda planta móvil se caracteriza por incluir una trituradora de impacto para la trituración secundaria, con capacidad máxima de 400 t/h, (En la FASE I tendrá una capacidad utilizada del $37 \%$ ).

- Las vías de acceso deben permitir la movilidad de los vehículos que transportan desde los puntos de transferencia cerrados y adecuados. Se recomienda para doble sentido una vía de $8,5 \mathrm{~m}$ de ancho (Para la Fase I, se contará con una flota de 20 vehículos con capacidad de $14 \mathrm{~m}^{3}$, con el fin de alcanzar la gestión de recolección diaria de $1.200 \mathrm{~m}^{3} /$ día. Éstos, realizarán entre 4 y 5 recorridos diarios ya que cada recorrido ida y vuelta dura en promedio un total de 1,5 horas tomando en cuenta el tiempo de carga y descarga, además de una holgura de 30 min. por imprevistos).

- Debe contarse con un área para la recepción de material de $3.000 \mathrm{~m}^{2}$ para almacenar aproximadamente $6.000 \mathrm{~m}^{3} \mathrm{de}$ RCD contando con la demanda diaria de $1.200 \mathrm{~m}^{3}$, teniendo en cuenta una hol- gura de hasta 5 días de almacenamiento por cualquier imprevisto.

- Para conocer las dimensiones óptimas del área de recepción de material o patio de maniobras y, suponiendo una relación de los lados 2:1, se utiliza la siguiente Ecuación Áurea de la Arquitectura, (2):

Ancho $o_{1}=\sqrt{\frac{3000 m^{2}}{2}}=38,7 m \approx 40 m$ (2)

- Por lo tanto el lado 2, Longitud sería dos veces la cantidad obtenida para el ancho, es decir un total de $80 \mathrm{~m}$.

- Una vez determinadas las dimensiones del Patio de Maniobras, el número de muelles se determinó por el modelo de Planeación de la Zona con Puerta Trasera, como se muestra en la Figura No. 5. Luego se propone incluir en el área 7 muelles, con una distancia entre los centros de las volquetas $(Y)$ de 4,0 m, con un ancho total (W) de $29,4 \mathrm{~m}$ y un largo total (L) de $18,5 \mathrm{~m}$.

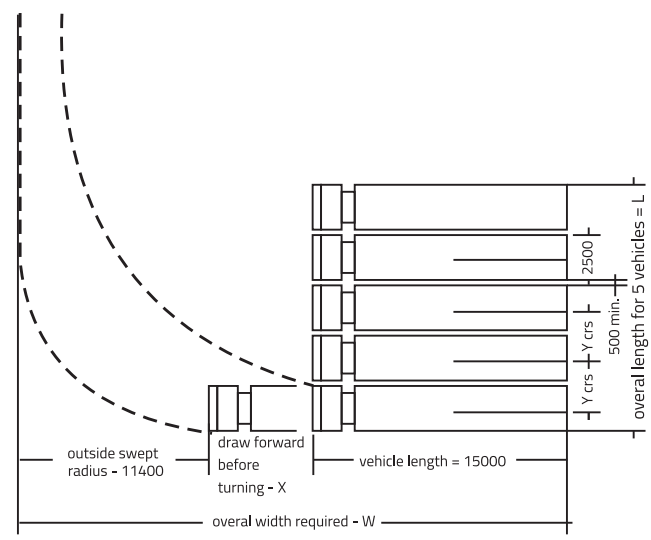

Figura 5. Modelo de Planeación de la Zona con Puerta Trasera para el diseño del Patio de Maniobras 
- Un sistema de ventilación adecuado, prevención y control de incendios; como también un sistema de drenaje para manejo de aguas provenientes de lluvias y de escorrentía por medio de la gravedad (aprovechamiento). Aspersores de agua, que permitan minimizar la cantidad de material particulado en las operaciones de trituración. Los aspersores deberán encontrarse a una distancia entre 8 y 12 $\mathrm{m}$, preferiblemente en las esquinas del área de trituración. Se eligió un Área de Selección Manual cubierta, 50 m², minimizando los riesgos para el personal que hará servicios de 8 horas en dos turnos.

\section{CONCLUSIONES}

Debido a que los RCD son materiales inertes generados en cada una de las epatas de la construcción civil, a lo largo de su vida útil (Aproximadamente cincuenta años), que constituyen del 41 al $60 \%$ de la cantidad total de los residuos generados en las ciudades y como poseen gran potencial de reincorporación en las cadenas productivas y económicas, son objeto de estudio y análisis a nivel nacional e internacional.

Comparando el nivel de tecnificación, se identifica la gran brecha que posee Colombia en relación a otros países, como España y Brasil, que cuentan actualmente con varias instalaciones de recuperación.

El País a la fecha cuenta con la única práctica de realizar vertido en áreas para recuperación, como también la ilegal en áreas públicas propuestas para otros menesteres; siendo escasa alguna otra alternativa de valorización, como el reuso o reciclaje, ya que menos del $5 \%$ son sometidos a estos procesos en su mayoría artesanales.

Razón por la cual, se propuso el diseño de la "Unidad Logística de Reciclaje Piloto de RCD para Bogotá D.C." como solución ecoeficiente, donde se obtuvo una cuantificación de los residuos generados, arrojando un resultado proyectado para el año 2012 de $14,230,405 \mathrm{~m}^{3}$, que después de ser depurado se obtuvo una cantidad objetivo a tratar de 8,181 m³/día, en la zona de influencia.

Según la caracterización y el análisis se estructuró el proceso de recuperación y reciclaje donde las actividades principales que lo componen son: Recepción del material, control y peso, separación magnética, clasificación primaria, por tamaños o granulométrica; separación neumática, y las operaciones de trituración primaria y secundaria.

Por facilidad del diseño se realizó la propuesta para tres fases de construcción y operación. La Fase I del proyecto, abarca inicialmente del 15\% aproximado de la cantidad de escombros total objetivo de la Unidad $\left(1,200 \mathrm{~m}^{3}\right)$; la Fase II procesará 3,200 $\mathrm{m}^{3} /$ día y finalmente, la Fase III completará el valor de 8,181 m²/día. Se eligió para la Fase I, una estructura tecnológica modular que consta de dos plantas móviles de trituración y una criba móvil. Se realizó la distribución en planta potencial que cumple con los parámetros de diseño de las unidades de trabajo y los requisitos mínimos de se- 
guridad industrial y salubridad, teniendo en cuenta áreas de almacenamiento y patios de maniobra.

La implementación de esta estructura en la ciudad evitará la sobreexplotación de áridos o agregados naturales y de canteras, obteniendo mayores rendimientos y consecuentemente, ahorro de energía y disminución de la contaminación atmosférica y del medio; dando solución al problema de los vertimientos ilegales que abundan en la ciudad. Uno de los resultados más significativos es la participación activa y el desarrollo de la comunidad, materia fundamental de la responsabilidad social, mediante la identificación e involucramiento de cada generador, lo que maximiza la contribución al desarrollo sostenible.

Los áridos obtenidos en este proceso deberán cumplir con características de calidad y costo competitivos en el mercado en donde serán usados; todo lo anterior, apoyado en este sistema logístico que genera valor agregado para los materiales antes de llegar al consumidor final.

\section{REFERENCIAS}

[1] Plan Nacional de Desarrollo Sostenibilidad Ambiental y Gestión del Riesgo. (2011). Departamento Nacional de Planeación. Colombia.

[2] Chávez Porras, A. (2007). Uso de lodo de estación para tratamiento de agua y agregado reciclado para la fabricación de elementos de albañilería [Tesis Doctoral]. Campinas, Brasil: Universidad Estatal de Campinas, Ingeniería Civil.

[3] Unidad Administrativa Especial de Servicios Públicos - UAESP. (2009). Diagnóstico del Manejo Integral de Escombros en Bogotá Distrito Capital. Bogotá.

[4] Mercante, I. Bovea, M. Arena, P. Martinengo, P. (2009). Estudio Comparativo de los Aspectos Técnicos entre la Legislación de RCD en España y América Latina. Barranquilla. Memorias II Simposio Iberoamericano de Ingeniería de Residuos. Barranquilla, 24 y 25 de septiembre de 2009. Red de Ingeniería en Saneamiento Ambiental y Universidad del Norte.

[5] Secretaria Distrital de Ambiente. Plan de Ordenamiento Territorial 2012 - 2016. (2012). Bogotá D.C.

[6] Toscano Machado, E. (2008). Gestión de la Ciencia y Tecnología para el Reciclado de los Desechos Sólidos en la Construcción [Tesis de Maestría en Gerencia de la Ciencia y la Innovación Tecnológica]. EMPROY Villa Clara. Cuba.

[7] Asociación Española de Gestores de Residuos de Construcción. (2012). Control de los Residuos de Construcción y Demolición, RCD, en los Ayuntamientos. Consultada en noviembre de 2012.

En: http://www.coaatba.es/colegio/descargas_del_editor/file/Cuadernillo_de_ Aytos.pdf. 
[8] Asesorías y Consultorías Técnicas y Ambientales - COAMBIENTE. (2009). Gestión Integral de Escombros. Anteproyecto para la Gestión Integral de los RC\&D en el Distrito Capital - Bogotá.

[9] Prieto García, F. Alonso Lavernia, J. Reciclaje de escombros de la construcción: Una alternativa ecológica para México. Revista AIDIS ARGENTINA. Ingeniería Sanitaria y Ambiental. Vol. 93. Pág. 93-101. Argentina.

[10] Defensoría del Pueblo. (2010). Situación actual del aprovechamiento de los residuos sólidos y la disposición final en el Relleno Sanitario Doña Juana en la ciudad de Bogotá D.C. Resolución Defensorial No. 61. Bogotá.

[11] Gobierno Nacional de Colombia. Decreto 357 de 1997 - Resolución Nº 541 de diciembre 14 de 1994.

[12] Gobierno de Chile. Agencia de Cooperación Internacional de Chile. (2012). Consultada en noviembre de 2012.

En: http://www.worldbank.org/depweb/ beyond/beyondsp/glossary.html.

[13] Cepeda, J. M. y Pizarro Gómez, A. (2012). Introducción al subsistema de producción. Departamento de Dirección de Empresas y Marketing. Universidad de Huelva. España.

[14] World Business Council for Sustainable Development. (2000). Ecoeficiencia,
Creando Más Valor con Menos Impacto. Consejo Empresarial Colombiano para el Desarrollo Sostenible.

[15] Alcaldía Mayor de Bogotá. Página web oficial de la Secretaría Distrital de Ambiente - SDA. Escombros, conceptos básicos. Coordinación de Infraestructura y Megaproyectos (2012). Consultada en agosto de 2012.

En: http://ambientebogota.gov.co/web/ escombros/conceptos-basicos..

[16] Instituto Colombiano de Normas Técnicas y Certificación - ICONTEC. (2008). Sistemas de Gestión de la Calidad. Requisitos. Tercera Actualización. Bogotá - Colombia.

[17] Instituto Colombiano de Normas Técnicas y Certificación - ICONTEC. (2004). Sistemas de Gestión Ambiental. Requisitos con Orientación para su Uso. Primera Actualización. Bogotá - Colombia.

[18] Instituto Colombiano de Normas Técnicas y Certificación - ICONTEC. (2007). Sistemas de Gestión en Seguridad y Salud Ocupacional. Requisitos. Primera Actualización. Bogotá - Colombia.

[19] Norma Internacional ISO 26000:2010. (2010). Guía de Responsabilidad Social: Primera Edición. Noviembre 01 de 2010.

[20] Instituto Colombiano de Normas Técnicas y Certificación - ICONTEC. (2011). Gestión del Riesgo. Principios y Directrices. Primera Actualización. Bogotá - Colombia. 
[21] Aneiros Rodríguez, L. (2008). Tecnología de las Plantas de Reciclaje de RCD y Niveles Tecnológicos en la UE. Revista Residuos No 103, marzo-abril 2008. Consultada en octubre de 2011.

En: http://www.infoambiental.es/html/ files/pdf/amb/iq/R103-56.pdf.

[22] Díaz A., Álvarez A., González M.J., (2004). Logística Inversa y Medio Ambiente. Editorial Mc Graw Hill. España.

[23] Velázquez Matretta, G. (1994). Administración de los Sistemas de Producción, Grupo Noriega Editores, México.

[24] Chávez A., Guarín N., Cortés M. (2013). Determinación de propiedades físico -químicas de los materiales agregados en muestra de escombros. Revista Ingenierías. Universidad de Medellín. Colombia. 2013, vol.12, n.22, pp. 45-58.

[25] Consejo Nacional de Medio Ambiente CONAMA. (2002). Resolución N³07, de 5 de julio de 2002. Brasil.

[26] Biurrarena. (2008). Planta de reciclaje de RCD mezclados y muy mezclados Ecovert, en la comarca del Bajo Cinca (Huesca). Reportaje. Consultado en marzo 2013. En: http://www.inese.es/html/ files/pdf/amb/iq/R106-18.pdf

[27] Gestión y Desarrollo del Medio Ambiente de Madrid - GEDESMA. (2013). Complejo de Tratamiento Integrado de Residuos de Construcción y Demolición de Navalcarnero. Madrid. Consultado en marzo 2013.
En: http://www.uteplantanavalcarnero. es/depósito_rechazo_planta.htm|\#

[28] Serrano, M. y Acosta, M. (2009). Evaluación Económica de una Microempresa de Reciclaje de Escombros. II Simposio Iberoamericano de Ingeniería de Residuos. Barranquilla, septiembre de 2009.

[29] Euroestudios - Ingenieros de Consulta. (2006). Planta de Tratamiento de RCD en Arganda del Rey. Arganda del Rey, Madrid.

[30] Tabernero, J.A. (2008). Planta de Tratamiento de Residuos de Construcción y Demolición de Gardelegui. $1^{\circ}$ Congreso Local: Empresas y Medio Ambiente, Miranda de Ebro.

[31] Gabinete de Estudios Ambientales y Agronómicos - G.A. INGENIEROS. (2009). Proyecto de Planta de Tratamiento de Residuos de Construcción y Demolición en Burgohondo.

[32] Alcaldía Mayor de Bogotá - Secretaria Distrital de Ambiente. (2009). Guía Ambiental para el Manejo Integral de Escombros en la Ciudad de Bogotá.

[33] Sánchez Mosquete, J. Plantas Recicladoras de RCD. (2010). Consultada en octubre de 2011.

En: http://issuu.com/jorgemosquete/ docs/plantas_reciclado_de_rcd.

[34] Wagner de Souza, J. Esguícero, F. J. y Luizmartinso, B. (2008). Implementação de usina para reciclagem de resíduos da 
construção civil (rcc) como ação para o desenvolvimento sustentável - estudo de caso. XXVIII Encontro Nacional De Engenharia De Produção. Rio de Janeiro.
[35] METSO (2010). Plantas móviles de trituración Serie Lokotrack para contratistas. Folleto 1127-06-10-ESBL/Tampere-Español. Finlandia. 